\title{
A Case of Bithemia: A Rare Complication of Transjugular Intraheptic Portosytemic Shunt
}

\author{
Michael Zhang, MS III, Michael A. Valentino, MD, PhD
}

\section{INTRODUCTION}

Transjugular intrahepatic portosystemic shunt (TIPS) is a common procedure used to alleviate the secondary effects of portal hypertension including uncontrolled variceal bleeding, refractory ascites, and hepatic pleural effusion (hydrothorax). There are several well-known complications of TIPS, including portosystemic encephalopathy, hemolytic anemia, hepatic ischemia, and stent thrombosis. ${ }^{1}$ In this case report we present a rare but serious complication of TIPS - bilhemia - in which bile escapes into the bloodstream through a fistula between the biliary tree and the hepatic venous system.

\section{CASE PRESENTATION}

A 56 year-old female with cirrhosis due to chronic hepatitis C presented to the hospital for a scheduled esophagogastroduodenoscopy (EGD) for evaluation and banding of esophageal varices. During the procedure, a large varix ruptured, requiring the patient to undergo an emergent TIPS procedure to control the hemorrhage.

A TIPS was attempted using a $10 \mathrm{~mm} \times 10 \mathrm{~mm}$ Viatorr covered stent. In the operative report, the interventional radiologist noted that multiple unsuccessful attempts were made accessing the right portal vein, requiring repeated repositioning of the cannula. Ultimately, a successful bridge was created between the right hepatic vein and central portal vein, which reduced the portosystemic gradient from $14 \mathrm{mmHg}$ to $3 \mathrm{mmHg}$. The patient was stabilized hemodynamically with a transfusion of seven units of packed red blood cells and was admitted to the medical intensive care unit (MICU).

Three days following the procedure the patient became jaundiced. Her laboratory profile at that time showed that her total and direct bilirubin levels had increased almost 10-fold, while her transaminases and INR remained stable (Table 1). An abdominal ultrasound was performed to evaluate the stent, which was found to be patent. There was also no evidence of biliary obstruction/dilatation on ultrasound. Over the course of her hospital stay, the patient's bilirubin continued to rise (Figure 1) while her alkaline phosphatase, transaminases, and INR remained stable (Figures 2-3). The continual rise in bilirubin prompted an abdominal CT, which showed a patent TIPS and no evidence of hepatic ischemia or biliary duct dilatation.

\section{DIFFERENTIAL DIAGNOSIS}

Elevated direct bilirubin levels are typically a sign of hepatocellular or biliary injury/obstruction. After undergoing TIPS placement, the shunting of blood from the portal vein to the systemic circulation can occasionally lead to hepatic ischemia. ${ }^{1}$ Clinically, this

Table 1. Liver function tests pre-TIPS and three days post-TIPS.

Antibiotic

Total bilirubin ( $\mathrm{mg} / \mathrm{dL})$

Direct bilirubin $(\mathrm{mg} / \mathrm{dL})$

Alkaline phosphatase (U/L)

AST (U/L)

$\operatorname{ALT}(U / L)$

INR
Pre-TIPS (normal range)

$1.4(0.1-0.9)$

$0.6(0.0-0.3)$

$58(25-120)$

$144(7-42)$

$37(1-45)$

$1.66(0.79-1.21)$

3 Days Post-TIPS

12.2

9.1

90

139

57

1.15 
presents as worsening right upper quadrant abdominal pain, hepatic encephalopathy, elevated aspartate transaminase (AST) and alanine transaminase (ALT) levels as well as an elevated serum bilirubin. ${ }^{1-2}$ However, the patient had an isolated hyperbilirubinemia, without transaminase elevation or worsening synthetic liver function. In addition, she had no abdominal pain and demonstrated no signs of encephalopathy. Therefore it was determined that hepatic ischemia was an unlikely diagnosis. To that same effect, worsening cirrhosis was equally unlikely as the patient demonstrated neither deterioration in her synthetic liver function nor any clinical signs of decompensated cirrhosis such as ascites or encephalopathy. Moreover, worsening cirrhosis post-TIPS is typically associated with TIPS thrombosis or stenosis, and both the ultrasound and CT scan showed a patent TIPS. 3

Biliary tree stricture or blockage is another condition that can lead to hyperbilirubinemia. Again, we considered this unlikely due to the patient's isolated hyperbilirubinemia, normal alkaline phosphatase level, as well as a lack of bile duct dilatation on ultrasound and CT scan.

Bilhemia is a rare condition which presents with sudden jaundice and a rapid elevation in direct bilirubin without evidence of hepatocellular injury or biliary obstruction. ${ }^{3}$ It was determined that this was the likely diagnosis given the patient's rapidly rising bilirubin levels in the setting of otherwise unchanged liver function tests and no evidence of obstruction on imaging. Furthermore, the biliary-venous fistula could be explained by the difficulty encountered in cannulating the portal vein during the TIPS procedure.

\section{OUTCOME AND FOLLOW-UP}

Fifteen days after the TIPS procedure, an ERCP was performed to evaluate for evidence of a biliary-venous fistula. The cholangiogram did not show evidence of a fistula or bile duct dilatation. However, a common bile duct stent was placed and sphincterotomy was performed in an attempt to lower the biliary pressure in order to reduce the flow of bile through a potentially unseen fistula. Despite this procedure, the patient's bilirubin continued to rise, and a venogram was performed to visualize and occlude the biliary-venous fistula. The venogram was also unremarkable, and it was determined that the only

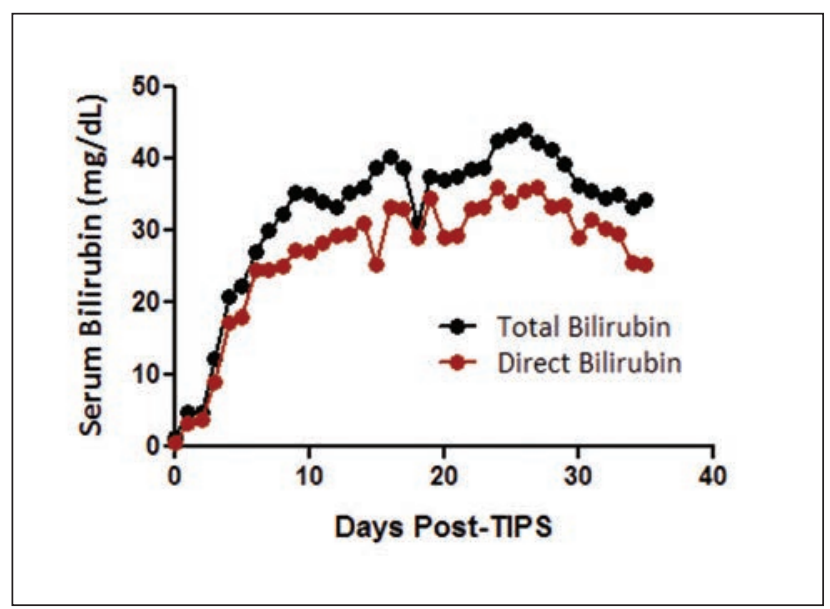

Figure 1. Total and direct serum bilirubin levels post-TIPS

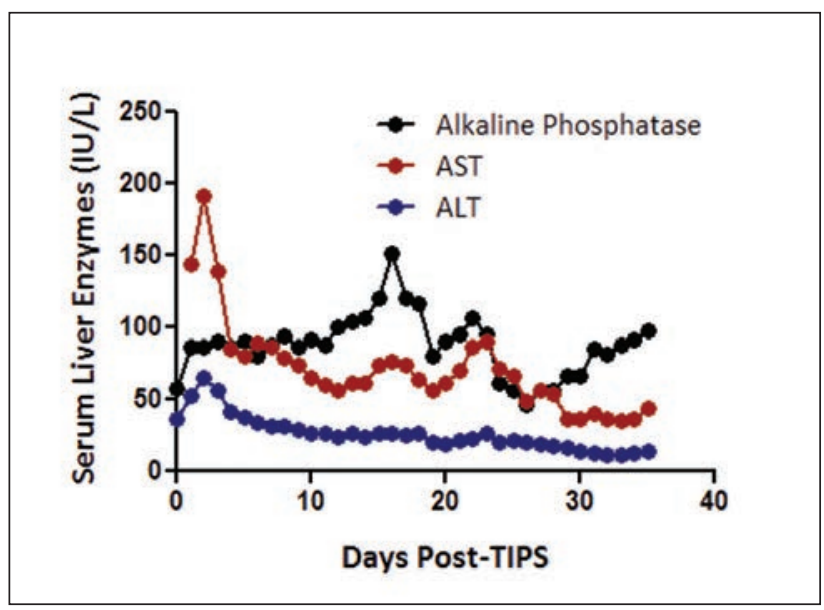

Figure 2: Serum alkaline phosphatase and hepatic transaminases post-TIPS

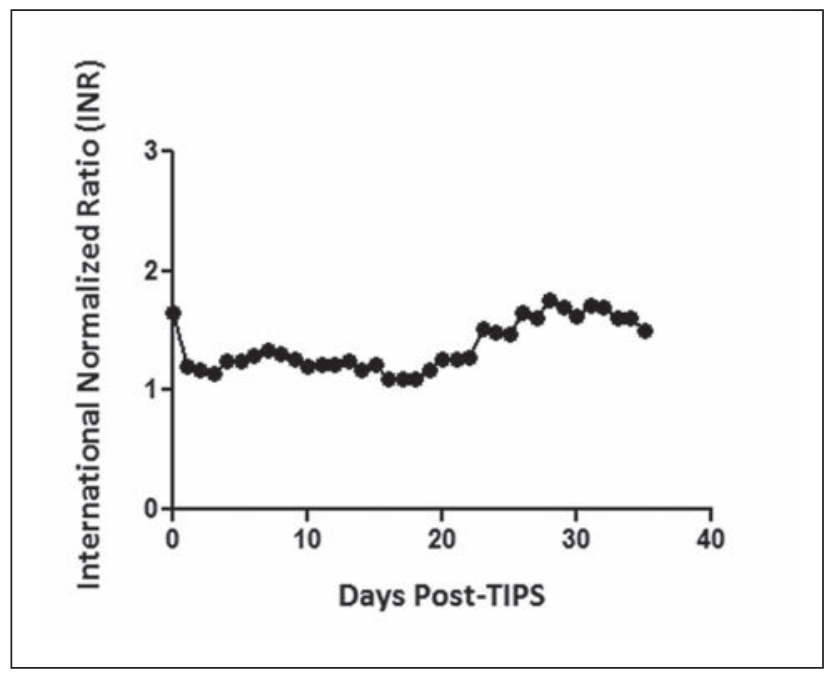

Figure 3: INR post-TIPS 
viable way to reverse the bilhemia was through liver transplantation.

During her hospital course, the patient developed a resistant Klebsiella bacteremia. Even with appropriate treatment, she remained persistently bacteremic, likely due to seeding of her TIPS. She developed sepsis complicated by renal failure and was transferred back to the MICU. Unfortunately, the patient's infection precluded her from undergoing liver transplantation and, after multiple discussions with the patient and her family, she was ultimately discharged home with hospice care.

\section{DISCUSSION}

Bilhemia is a condition characterized by a rapid rise in total and direct serum bilirubin without other signs of hepatic dysfunction or biliary obstruction. ${ }^{4}$ It is typically a consequence of hepatic trauma, though it has been known to occur as a complication of TIPS procedures. ${ }^{5}$ The pathophysiology relates to a pressure gradient between the common bile duct (mean pressure $=12-14$ $\mathrm{mmHg}$ ) and the hepatic vein (mean pressure $=7 \mathrm{mmHg}$ ) which results in the direct flow of bile into the hepatic vein. ${ }^{4,6}$ This is in contrast to a fistulous communication between the higher pressure portal venous system and the biliary tree which typically results in hemobilia (flow of blood into the biliary system).

One consequence of bilhemia is fat embolism from the passage of large amounts of undissolved bile into the systemic circulation. ${ }^{6-7}$ Bacteremia is another consequence and is thought to be caused by the bilious contamination of the systemic circulation with enteric flora. ${ }^{8-10}$

Treatments for bilhemia aim to decompress the biliary system, commonly with biliary stenting and sphincterotomy. ${ }^{6-8}$ Other case studies have shown that percutaneous biliary drainage, endoscopic nasobiliary drainage, and venous balloon occlusion are also viable options with liver transplantation being the only definitive treatment for bilhemia. ${ }^{4-5,7-8}$ In the absence of treatment, spontaneous closure of the biliary-venous fistula within three weeks has been observed.?

The patient's sudden jaundice and rapid rise in bilirubin three days after TIPS matches the clinical presentation of other cases reports of TIPS-induced bilhemia. In this case, biliary stenting and sphincterotomy was unsuccessful in managing the condition. Liver transplantation was determined to be the only option for treatment; however the patient's persistent bacteremia eliminated the possibility of transplant.

\section{KEY POINTS}

Bilhemia is a rare complication of TIPS which should be suspected in post-TIPS patients with a rapid rise in serum bilirubin without other signs of hepatic dysfunction. The formation of a biliary-venous fistula can lead to thromboembolism and bacteremia. Decompression of the biliary tree should be attempted, and liver transplantation should be prioritized as this condition is fatal in $\sim 50 \%$ of cases. ${ }^{6}$

\section{REFERENCES}

1. Freedman AM, Sanyal AJ, Tisnado J, et al. Complications of transjugular intrahepatic portosystemic shunt: a comprehensive review. Radiographics. 1993;13: 1185

2. Gaba RC, Khiatani VL, Knuttinen MG et al. Comprehensive review of TIPS technical complications and how to avoid them. American Journal of Roentgenology. 2011;196: 675-685

3. Han G, Qi X, He C et al. Transjugular intrahepatic portosystemic shunt for portal vein thrombosis with symptomatic portal hypertension in liver cirrhosis. J Hepatol. 2011; 54(1): 78-88.

4. Kruel CR, Guimarães M, Chedid AD, et al. Bilhemia following transjugular intrahepatic portosystemic shunt placement (TIPS): liver transplantation as a rescue procedure - case report. Arq Bras Cir Dig. 2013;26(3): 238-40.

5. Blum U, Buitrago-Tellez C, el Seif M, et al. Posttraumatic bilhemia: conservative management by percutaneous drainage. Cardiovasc Intervent Radiol. 1993;16(1): 55-7.

6. Singal AK, Kathuria MK, Malhotra A et al. Bithemia after trans-jugular intra-hepatic porto-systemic shunt and its management with biliary decompression. World J Gastroenterol. 2009;15(29):3681-3.

7. Spahr L, Sahai A, Lahaie R et al. Transient healing of TIPS-induced biliovenous fistula by PTFE-covered stent graft. Dig Dis Sci. 1996; 41(11): 2229-32.

8. Mallery S, Freeman ML, Peine CJ et al. Biliary-shunt fistula following transjugular intrahepatic portosystemic shunt placement. Gastroenterology. 1996;111(5): 1353-7.

9. Jawaid Q, Saeed ZA, Di Bisceglie AM, et al. Biliary-venous fistula complicating transjugular intrahepatic portosystemic shunt presenting with recurrent bacteremia, jaundice, anemia and fever. Am J Transplant. 2003;3(12): 1604-7.

10. Willner IR, El-Sakr R, Werkman RF, et al. A fistula from the portal vein to the bile duct: an unusual complication of tranjjugular intrahepatic portosystemic shunt. Am J Gastroenterol. 1998;93(10): 1952-5. 